\title{
Fatores associados à internação hospitalar em mulheres na pós-menopausa: Um estudo de coorte no município de Santa Maria/RS
}

\author{
Tamires Tolazzi Martins ${ }^{1}$; Ana Elize Barin² ; Raisa Bringhenti ${ }^{3}$; \\ Adhan Rizzi de Vieira ${ }^{4}$; Fabio Vasconcellos Comim $^{5}$ e Melissa \\ Orlandin Premaor6.
}

\section{Resumo}

INTRODUÇÃO: Considerando o fato de que pacientes frágeis internam com mais frequência que outros, observou-se que as internações hospitalares podem ser importantes marcadores de morbidade da população. Dessa forma, buscou-se avaliar nesse estudo os fatores de risco associados à internação hospitalar que auxiliam na identificação desses pacientes. Assim, o objetivo do estudo em questão foi identificar os fatores associados à internação hospitalar em mulheres na pós-menopausa atendidas na atenção primária do município de Santa Maria.

MÉTODOS: Um estudo de coorte foi realizado nas unidades básicas de saúde do município de Santa Maria. Nesse estudo foi aplicado um questionário padronizado que incluiu detalhes sobre a história médica pregressa de todas as mulheres que aceitaram participar do estudo. $\mathrm{O}$ seguimento foi realizado por contato telefônico um ano após o recrutamento das participantes. Considerou-se internação hospitalar a permanência igual ou superior a 24 horas em um hospital ou serviço de pronto atendimento do município.

RESULTADOS: Das 1057 mulheres recrutadas para o estudo, 799 destas completaram o primeiro seguimento. Verificou-se que a média de idade dessas mulheres era de 67 anos (Desvio Padrão 7,5) e constatou-se 
também que 69,4\% delas apresentavam pelo menos uma comorbidade. Dentre as comorbidades analisadas, a presença de hipotireoidismo foi o único fator associado à internação hospitalar [razão de chances 1,7 (Intervalo de Confiança 1,1 a 2,7; $p$ valor $=0,012)]$. Apesar de ter permanecido no modelo de análise multivariada o uso dos braços para levantar da cadeira não foi estatisticamente significativo [razão de chances 0,7 (Intervalo de Confiança 0,4 a 1,$0 ; P=0,064)]$.

CONCLUSÕES: Logo, a presença de hipotireoidismo não-tratado parece estar associada à internação hospitalar em mulheres na pósmenopausa, participantes do estudo, atendidas na atenção primária de saúde.

Palavras Chave: internações; comorbidade; mulheres 
${ }^{1}$ Graduanda do curso de Medicina da Universidade Federal de Santa Maria (UFSM), membro do grupo de pesquisa de Doenças Endócrino-Metabólicas Prevalentes e bolsista do Programa de Iniciação Científica para o Hospital Universitário de Santa Maria PROIC-HUSM; tamitolazzi@gmail.com

${ }^{2}$ Graduanda do curso de Medicina da Universidade Federal de Santa Maria (UFSM) e membro do grupo de pesquisa de Doenças Endócrino-Metabólicas Prevalentes; anaelizebarin@gmail.com

${ }^{3}$ Graduanda do curso de Medicina da Universidade Federal de Santa Maria (UFSM), membro do grupo de pesquisa de Doenças Endócrino-Metabólicas Prevalentes e bolsista do Programa de Iniciação Científica para o Hospital Universitário de Santa Maria PROIC-HUSM; br.raisa@gmail.com

${ }^{4}$ Graduando do curso de Medicina da Universidade Federal de Santa Maria (UFSM), membro do grupo de pesquisa de Doenças Endócrino-Metabólicas Prevalentes e bolsista de Iniciação Científica - PROBIC-FAPERGS. adrivsama@gmail.com

${ }^{5}$ Pesquisador do grupo de pesquisa de Doenças Endócrino-Metabólicas Prevalentes e Orientador do Programa de Iniciação Científica para o Hospital Universitário de Santa Maria - PROIC-HUSM. fabio.comim@bol.com.br

${ }^{6}$ Pesquisadora do grupo de pesquisa de Doenças Endócrino-Metabólicas Prevalentes e Orientadora do Programa de Iniciação Científica para o Hospital Universitário de Santa Maria - PROIC-HUSM. premaor@ufsm.com.br 